\title{
Aspirin "Allergy"-Induced Thrombocytopenia: A Case Report
}

This article was published in the following Dove Press journal:

Journal of Asthma and Allergy

\section{Wei Fu \\ Shanshan Xu \\ Xiang Dong \\ Qing Xie (D) \\ Yadong Gao (1D)}

Department of Allergology, Zhongnan Hospital of Wuhan University, Wuhan, Hubei, People's Republic of China
Correspondence: Yadong Gao; Wei Fu Department of Allergology, Zhongnan Hospital of Wuhan University, Wuhan, Hubei, 43007I, People's Republic of China Tel +86 I507I384536

Email gaoyadong@whu.edu.cn; fuwei05II@I63.com

\begin{abstract}
Aspirin is clinically widely used to inhibit platelet aggregation after coronary intervention. Herein we describe a case of aspirin-induced thrombocytopenia that may be related to allergy to aspirin. A 47-year-old man developed a delayed hypersensitivity reaction to aspirin, with pruritus, purpura and thrombocytopenia, increased peripheral blood eosinophils and enlarged inguinal lymph node. All the symptoms disappeared in 2 years after stopping aspirin. Aspirin-induced thrombocytopenia related to allergy is rarely reported. Aspirin hypersensitivity should be taken into consideration in case of unexplained thrombocytopenia in patients taking aspirin. Aspirin "allergy"-induced thrombocytopenia may involve both aspirin related $\mathrm{IgG}$ and $\mathrm{IgE}$ antibodies.
\end{abstract}

Keywords: aspirin, allergy, thrombocytopenia, platelets

\section{Introduction}

Aspirin has been widely used in patients with coronary heart disease to reduce morbidity and mortality. ${ }^{1}$ Aspirin can inhibit platelet aggregation by inhibiting the activity of COX (cyclooxygenase)-1 and then reduce the synthesis of TXA2 (thromboxane A2). ${ }^{2}$ Drug hypersensitivity reactions caused by aspirin include aspirinexacerbated respiratory disease or cutaneous disease associated with COX-1 inhibition, aspirin-induced urticaria/Angioedema or anaphylaxis mediated by specific IgE, and aspirin-induced delayed hypersensitivity reactions ${ }^{3}$. Most of aspirin-induced delayed hypersensitivity reactions are severe, such as DIHS/DRESS (drug-induced hypersensitivity syndrome/drug reaction with eosinophilia and systemic symptoms), AGEP (acute generalized exanthematous pustulosis), and less frequent TEN/SJS (StevensJohnson syndrome/toxic epidermal necrolysis). ${ }^{4}$ Mechanically, aspirin-induced delayed hypersensitivity is T cell-dependent. ${ }^{5}$ Aspirin-induced thrombocytopenia has been rarely reported. ${ }^{6}$ Herein, we report a case of aspirin-induced thrombocytopenia, which might be associated with allergy to aspirin.

\section{Case Report}

A 47-year-old male patient presented with recurrent thrombocytopenia with general pruritus and increased peripheral blood eosinophil count for 2 years. The patient received coronary stent implantation due to acute coronary syndrome on September 1, 2014. After that he took aspirin $100 \mathrm{mg} / \mathrm{d}$, clopidogrel $75 \mathrm{mg} / \mathrm{d}$ and Lipitor $10 \mathrm{mg} / \mathrm{d}$ regularly. Recurrent systemic ecchymosis and pruritus occurred, but he did not ask for medical consultant since the symptoms were mild.Pruritus and ecchymosis persisted after discontinuing clopidogrel, which will be routinely 
stopped 1 year after the intervention. He was not reported other underlying diseases and related family history except a right inguinal mass, which was excised and diagnosed as angiolymphoid hyperplasia with eosinophilia (ALHE) in 2008. In September 2015, that the patient found a lump at right groin, which were confirmed to be enlarged bilateral inguinal lymph nodes with ultrasound examination. Blood routine test indicated normal platelet count $\left(244 \times 10^{9} / \mathrm{L}\right)$ but with decreased lymphocyte count and increased basophil count. The patient continued the treatment of aspirin. In June 2016, the pruritus became severe, and the eosinophil percentage was $17.6 \%\left(1.47 \times 10^{9} / \mathrm{L}\right)$, and the platelet count was $151 \times 10^{9} / \mathrm{L}$. No significant change was observed in a repeat test after one week. The patient refused morphological examination of the peripheral blood and only took antihistamines to control pruritus. On November 2, 2016, a large amount of ecchymosis appeared on the limbs. Blood eosinophil percentage increased to $18.2 \%$, and the platelet count decreased to $36 \times 10^{9} / \mathrm{L}$. The patient was admitted into hospital. Tests for platelet antibody (PAIgM+: $39.2 \%$, PAIgA+: $16.96 \%$ ) and platelet specific antibodies (platelet IIb/IIIa and Ia/IIa antibodies) were positive. Bone marrow cytology, bone marrow immunotyping and bone marrow chromosome were all in normal range, and bone marrow PDGFR fusion gene was negative. The patient was treated with intravenous glucocorticoids and sequentially treated with oral prednisone. He was discharged after the ecchymoses improved and the platelet and eosinophil counts returned to the normal range. Ultrasound examination showed the inguinal lymph nodes decreased to normal size. Oral prednisone was withdrawn 2 months later. After that, a small amount of ecchymoses and enlarged inguinal lymph nodes reoccurred, and then the patient adjusted the doses of aspirin and prednisone according to the symptoms and platelet.

In May 2018, the patient came to our department for his pruritus. The level of total IgE was $1233 \mathrm{kU} / \mathrm{L}$ (normal range: $<60 \mathrm{kU} / \mathrm{L}$ ); the skin prick test for common allergens and patch test for various metals were negative, and serum specific IgE against common allergens were all in normal range (Figure S1). On June 17, 2018, the patient was admitted to our hospital due to thrombocytopenia $\left(15 \times 10^{9} / \mathrm{L}\right)$, massive ecchymosis and severe pruritus. After transfusion with platelets and immunoglobulin gamma, the platelet count returned to the normal range. However, a more severe thrombocytopenia $\left(9 \times 10^{9} / \mathrm{L}\right)$ and ecchymosis with pruritus occurred one month later. The symptoms was improved after treatment with intravenous
Table I Prednisone Low Dose Maintenance Regiment

\begin{tabular}{|l|l|l|}
\hline Dose (mg) & Frequency & Duration \\
\hline 10 & qd & Two weeks \\
5 & qd & Two weeks \\
2.5 & qd & One month \\
2.5 & qod & One month \\
\hline
\end{tabular}

Abbreviations: TXA2, thromboxane A2; COX-I, cyclooxygenase-I; DIHS/ DRESS, drug-induced hypersensitivity syndrome; AGEP, acute generalized exanthematous pustulosis; TEN/SJS, Stevens-Johnson syndrome/toxic epidermal necrolysis; ALHE, angiolymphoid hyperplasia with eosinophilia.

glucocorticoids and transfusion of platelets. After discharge, the patient continued taking low doses of oral prednisone and the regimen was shown in Table 1.

After detailed history inquiry and retrospectively analyzed the longitudinal variations of blood routine tests, we noticed that the thrombocytopenia always occurred several days after intake of aspirin, and the platelet count rose several days after withdrawal of aspirin. In consideration of a possible allergic reaction to aspirin, he was suggested to stop aspirin. After discontinuing aspirin from July 2018, he did not present with ecchymosis and pruritus anymore in the next two years. The blood platelet count was kept $>$ $200 \times 10^{9} / \mathrm{L}$ by monthly monitoring, and the eosinophil counts gradually returned to normal range. The enlarged right inguinal lymph nodes disappeared. The level of total IgE decreased to $159 \mathrm{kU} / \mathrm{L}$ after one year, and now is close to normal range $(67.5 \mathrm{kU} / \mathrm{L})$ (Figure S2)

\section{Discussion}

Drug-induced thrombocytopenia is not uncommon and can be induced by heparin, ceftriaxone, and some kinds of monoclonal antibodies, etc. ${ }^{7}$ It should be distinguished from idiopathic thrombocytopenic purpura. The temporal relationship between drug intake and thrombocytopenia and between drug withdrawal and the recovery of platelet count and symptoms are hints for the diagnosis of drug-induced thrombocytopenia. In this case, the patient underwent extensive tests to rule out other causes of thrombocytopenia in the first hospitalization.

The patient had been taking aspirin, clopidogrel and Lipitor after coronary stent implantation, and the platelet count was reviewed regularly every month after the intervention. The platelet count maintained at $(200-240) \times 10^{9} / \mathrm{L}$ at first year after the intervention (Figure S3). Except mild pruritus and subcutaneous bleeding, no other symptoms were complained by the patient. Nevertheless, the presence of pruritus indicates the possibility of allergy. The pruritus 
and subsequent thrombocytopenia should not be caused by clopidogrel, since the patient's symptoms worsened even after withdrawing of clopidogrel in the first year after the intervention. Lipitor also should not be the responsible medication for thrombocytopenia, because there was no temporal correlation between the intake of Lipitor and the occurrence of thrombocytopenia; and more importantly, the patient had never stopped taking Lipitor. Even after the mild decrease of platelet count $\left(151 \times 10^{9} / \mathrm{L}\right)$ and pruritus, the patient continued to take aspirin $100 \mathrm{mg} / \mathrm{d}$ until illness worsened 5 months later, with the platelet count of $36 \times 10^{9} / \mathrm{L}$. A series of tests were performed to find out possible causes for thrombocytopenia, however, hypersensitivity to aspirin was not suspected until consulting us. The temporal association between the decreased platelet count and aspirin intake, and the recovery of platelet count after aspirin discontinuation (Figure 1), as well as the eosinophil counts (Figure 2) fluctuated inversely with the platelet counts suggested the possibility of aspirininduced thrombocytopenia.

Drug-induced thrombocytopenia were divided into three types according to possible mechanisms: marrow suppression, immunological reactions, and nonimmunological thrombocytopenia. ${ }^{8}$ In this case, the thrombocytopenia appeared to be an immunological reaction due to the presence of platelet specific antibodies and absence of any evidence of bone marrow suppression. The immunological mechanisms underlying drug-induced thrombocytopenia can be divided into six types according to the types of

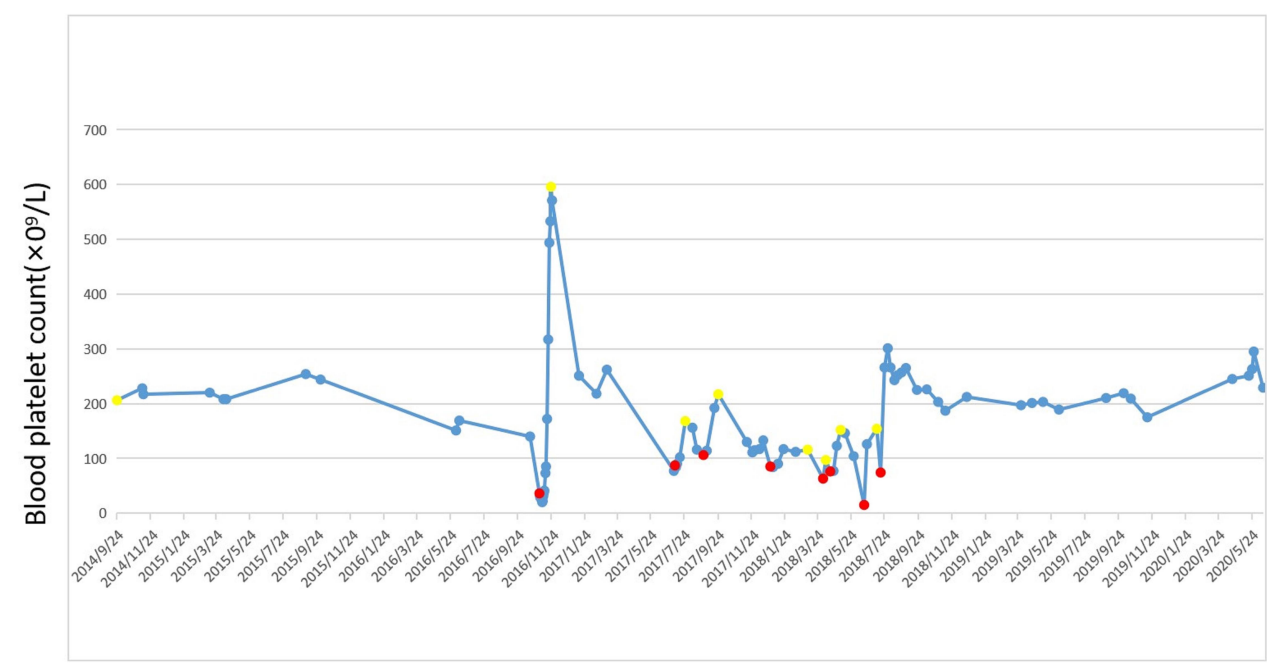

Figure I Fluctuation trend of platelet count after coronary stenting. Solid dots indicate the patient's platelet count on that day. The red dots indicate that the patient's platelet count and aspirin is discontinued on that day. The yellow dots indicate that the patient's platelet count and aspirin is taking on that day.

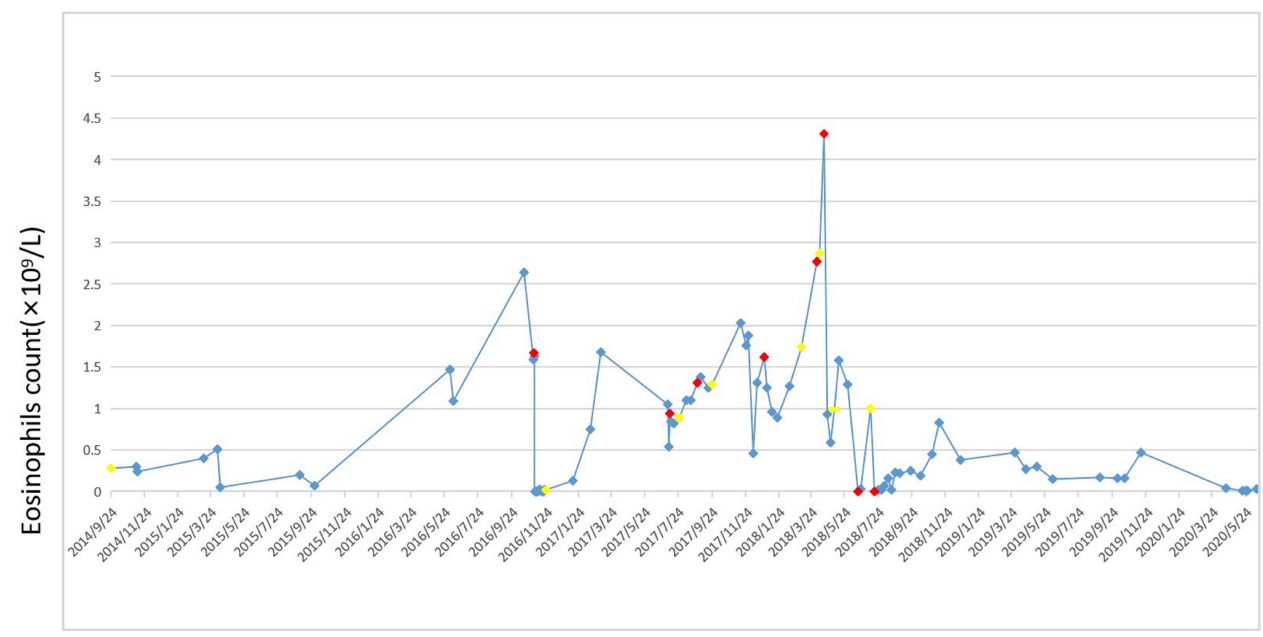

Figure 2 Fluctuation trend of eosinophils after coronary stenting. Solid rhombus indicate the patient's eosinophils count on that day. The red rhombus indicate that the patient's eosinophils count and aspirin is discontinued on that day. The yellow rhombus indicate that the patient's platelet count and aspirin is taking on that day. 


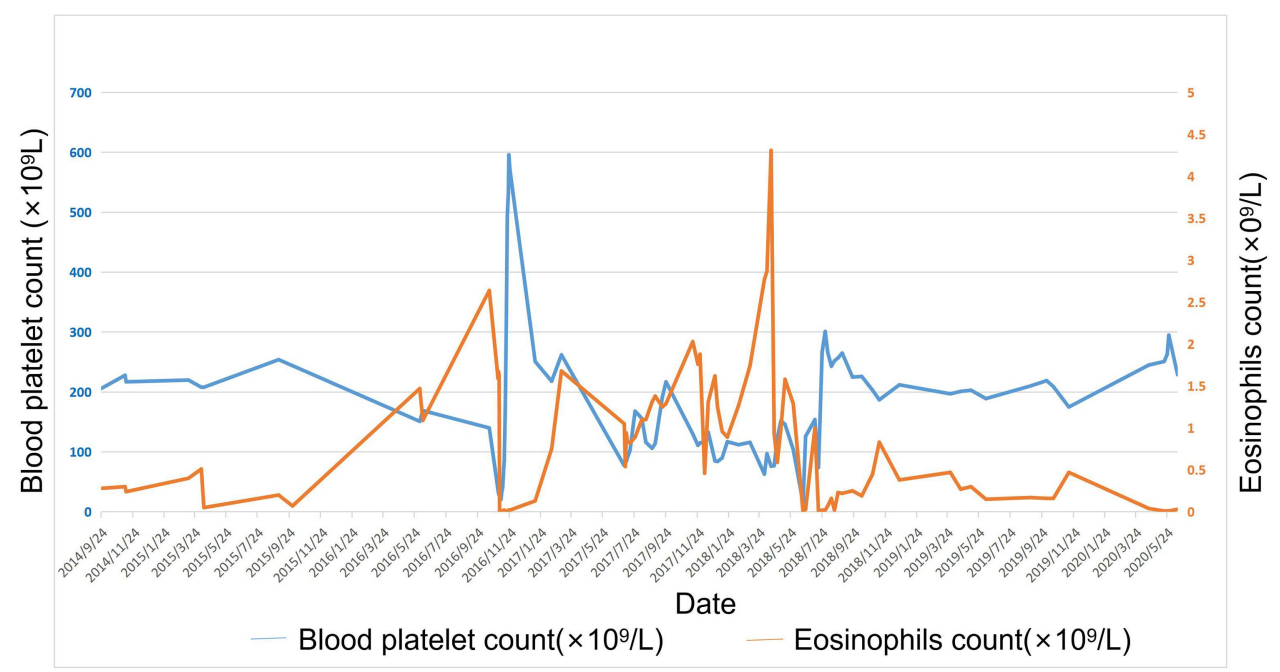

Figure 3 Fluctuation trend of platelets and eosinophils after coronary stenting.

antigens. ${ }^{9}$ In the case, the mechanisms underlying the immune impairment induced by platelet IIb/IIIa and Ia/ IIa antibodies (IgG) was not clear. We speculate that aspirin, a soluble drug, induced the production of plateletspecific antibodies, namely platelet IIb/IIIa and Ia/IIa antibodies, and accelerated the clearance of platelets and thus leading to thrombocytopenia. ${ }^{10}$ In addition, the total $\mathrm{IgE}$ level (aspirin-specific IgE test is not available in China) also fluctuated inversely to the platelet counts, which also indicates a possible involvement of Type I hypersensitivity, which may contribute to pruritus. Moreover, the eosinophil counts fluctuated inversely to the platelet counts (Figure 3) and returned to normal gradually after discontinuation of aspirin. All of these manifestations implied an immunological reaction to underlying thrombocytopenia. It is noticeable that there was a possible correlation between ALHE and thrombocytopenia in this patient in consideration of the temporal match. However, the exact causality is still unknown.

Several clinical presentations in this patient, such as pruritus, lymphadenopathy, and eosinophils, are similar to those of DRESS. By careful comparison, with the clinical and laboratory manifestations in this was not consistent with diagnostic criteria for DRESS. During the disease course, the patient presented neither with fever, liver or kidney disfunction, nor any signs of lung or other visceral involvement. Therefore, DRESS could be ruled out in this case.

\section{Conclusion}

When thrombocytopenia occurs in a patient intaking of aspirin, the possibility of aspirin-induced thrombocytopenia should be considered. Detailed clinical manifestation and history, and also laboratory findings will give clue to the diagnosis of this disease. The underlying mechanisms of aspirininduced thrombocytopenia are still not clarified, however, It may contributes to the immunological impairment, specially may involve in both aspirin related $\operatorname{IgG}$ and $\operatorname{IgE}$ antibodies.

\section{Consent for Publication}

Written informed consent for publication was obtained from the patient. The patient was informed that deidentified data would be used in scientific research and publications.

\section{Disclosure}

The authors have no conflicts of interest to declare.

\section{References}

1. Bittl JA, Baber U, Bradley SM, et al. Duration of dual antiplatelet therapy: a systematic review for the 2016 ACC/AHA guideline focused update on duration of dual antiplatelet therapy in patients with coronary artery disease: a report of the American College of Cardiology/American Heart Association Task Force on Clinical Practice Guidelines. J Am Coll Cardiol. 2016;68(10):1116-1139. doi:10.1016/j.jacc.2016.03.512.

2. Patrono C, Morais J, Baigent $\mathrm{C}$, et al. Antiplatelet agents for the treatment and prevention of coronary atherothrombosis. $\mathrm{J} \mathrm{Am} \mathrm{Coll}$ Cardiol. 2017;70(14):1760-1776. doi:10.1016/j.jacc.2017.08.037.

3. Kowalski ML, Asero R, Bavbek S, et al. Classification and practical approach to the diagnosis and management of hypersensitivity to nonsteroidal anti-inflammatory drugs. Allergy. 2013;68 (10):1219-1232. doi:10.1111/all.12260.

4. Plaza-Serón MDC, García-Martín E, Agúndez JA, et al. Hypersensitivity reactions to nonsteroidal anti-inflammatory drugs: an update on pharmacogenetics studies. Pharmacogenomics. 2018;19 (13):1069-1086. doi:10.2217/pgs-2018-0079. 
5. Roychowdhury S, Svensson CK. Mechanisms of drug-induced delayed-type hypersensitivity reactions in the skin. AAPS J. 2005;7 (4):E834-E846. doi:10.1208/aapsj070480.

6. Garg SK, Sarker CR. Aspirin-induced thrombocytopenia on an immune basis. Am J Med Sci. 1974;267(2):129-132. doi:10.1097/ 00000441-197402000-00008.

7. Mitta A, Curtis BR, Reese JA, et al. Drug-induced thrombocytopenia: 2019 Update of clinical and laboratory data. Am J Hematol. 2019;94 (3):E76-E78. doi:10.1002/ajh.25379.
8. Kenney B, Stack G. Drug-induced thrombocytopenia. Arch Pathol Lab Med. 2009;133(2):309-314. doi:10.1043/1543-2165-133.2.309.

9. Vayne C, Guéry E-A, Rollin J, et al. Pathophysiology and diagnosis of drug-induced immune thrombocytopenia. J Clin Med. 2018;19 (13):2212. doi:10.2217/pgs-2018-0079

10. Aster RH, Bougie DW. Drug-induced immune thrombocytopenia. $N$ Engl J Med. 2007;357(6):580-587. doi:10.1056/NEJMra066469.

\section{Publish your work in this journal}

The Journal of Asthma and Allergy is an international, peer-reviewed open-access journal publishing original research, reports, editorials and commentaries on the following topics: Asthma; Pulmonary physiology; Asthma related clinical health; Clinical immunology and the immunological basis of disease; Pharmacological interventions and new therapies. The manuscript management system is completely online and includes a very quick and fair peer-review system, which is all easy to use. Visit http://www.dovepress.com/testimonials.php to read real quotes from published authors.

Submit your manuscript here: https://www.dovepress.com/journal-of-asthma-and-allergy-journal 